\title{
Bridging Oxidase- and Oxygen Reduction Electro-catalysis by Model Single-atom Catalysts
}

Xiangyu Lu (逯向雨 $)^{1,2}$, Shanshan Gao (高珊珊) $)^{3}$, Han Lin $(\text { 林翰 })^{1}$, Han Tian (田 汉 $)^{1,2}$, Deliang Xu (徐德良) $)^{1}$, and Jianlin Shi (施剑林) ${ }^{1 *}$

${ }^{1}$ State Key Lab of High Performance Ceramics and Superfine Microstructure, Shanghai Institute of Ceramics, Chinese Academy of Sciences, Shanghai 200050, P. R. China.

${ }^{2}$ Center of Materials Science and Optoelectronics Engineering, University of Chinese Academy of Sciences, Beijing 100049, P. R. China.

${ }^{3}$ School of Public Health, Shanghai Jiao Tong University School of Medicine, Shanghai 200025, P. R. China.

E-mail: jlshi@mail.sic.ac.cn 
Abstract: Nanocatalysts with enzyme-like catalytic activities, such as oxidase-mimics, are extensively used in biomedicine and environmental treatment. Searching enzymelike nanomaterials, clarifying the origins of catalytic activity and developing activity assessment methodologies are therefore of great significance. Here we report that the oxidase- and oxygen reduction reaction (ORR) electro-catalysis can be well-bridged based on their identical activity origins, which makes the facile electrocatalytic ORR activity measurements intrinsically applicable to the oxidase-like activity evaluations. Inspired by natural heme-copper oxidases, $\mathrm{Cu} / \mathrm{Fe}$ doped single-atom catalysts (SACs) were first synthesized and used as model catalysts. Chromogenic reactions, electrochemical voltammetric measurements and density functional theory calculations further verify the linear relationship between their oxidase-like and ORR catalytic activities of the catalysts, thus an effective descriptor $\left(\left|\overline{j_{n}}\right|\right)$ are proposed for the rapid enzymic catalyst evaluation. The enhanced tumour therapeutic efficacy of SACs has been evidenced to result from their oxidase-like/ORR activities, which prove that numerous ORR electrocatalysts are promising candidates for oxidase mimics and tumour therapy. The synergistic catalytic effect of the biomimetic hetero-binuclear $\mathrm{Cu}$ Fe centres has also been probed thoroughly.

Keywords: Nanocatalytic medicine; Tumour therapy; Oxygen reduction electrocatalysts; Oxidases; Single atom catalysts

Nanomaterials with intrinsic enzyme-like characteristics show broad application potentials (for example, biosensors, immunoassays, disease diagnosis, medicine 
development, antifouling and environment treatment) owing to their high stability, low cost, tunability and high catalytic activities ${ }^{[1-4]}$. Among them, oxidases can catalyse the oxidation of organic molecules using $\mathrm{O}_{2}$, in which $\mathrm{O}_{2}$ is concurrently reduced to water, hydrogen peroxide or functional groups ${ }^{[4-7]}$. Catalysing the oxidation of biomolecules by oxidizing species such as $\mathrm{O}_{2}$ and $\mathrm{H}_{2} \mathrm{O}_{2}$ forms the basis of diverse biomedical applications, such as tumour therapy and antibiosis ${ }^{[8-10]}$. Unfortunately, $\mathrm{H}_{2} \mathrm{O}_{2}$ is generated from $\mathrm{O}_{2}$ in a kinetically slow manner in the preceding metabolic processes, resultantly its in vivo level is usually below a certain threshold, so the therapeutic outcomes by using $\mathrm{H}_{2} \mathrm{O}_{2}$ as substrate are undoubtedly limited ${ }^{[11,12]}$. Based on the principle of direct matter conversion by using $\mathrm{O}_{2}$ as the reaction substrate, oxidases are thus the most promising candidates for increasing the oxidative stress thanks to the sustainable supply of $\mathrm{O}_{2}$ from the blood circulation and their higher utilization efficiency of $\mathrm{O}_{2}$ than $\mathrm{H}_{2} \mathrm{O}_{2}$-involved pathway. Recent studies have found the oxidaselike properties of a number of electro-catalysts that have been used in oxygen reduction reactions $^{[8,13]}$, while some natural oxidases have also been explored as oxygen reduction electrocatalysts ${ }^{[14-16]}$. Hence, we hypothesize that the oxidase-like activities of catalysts are in accordance with their oxygen reduction reaction (ORR) activities in terms of their homology in activity origins. If this hypothesis is tenable, we could efficiently assess the oxidase-like activity of catalysts based on mature and rather facile voltammetric techniques, and great numbers of known oxygen reduction electrocatalysts, which have been widely employed in fuel cells, dye-sensitized solar 
cells, and metal-air batteries ${ }^{[17,18]}$, may have oxidase-like activities and could be further applied in other fields.

To verify this hypothesis, single-atom catalysts (SACs), in which single metal atoms as catalytically active sites are individually dispersed and constrained on the support, were chosen as the model catalysts. SACs are a kind of ideal heterogeneous catalysts featuring the maximized utilization of metal atoms while minimized ion cytotoxicity without the free metal ion release into the media ${ }^{[19]}$. Additionally, the welldefined and uniform structure of the catalytic site provides an ideal model system for the overall understanding of the reaction mechanisms at molecular level ${ }^{[20-22]}$. More importantly, the catalytic activity of SACs originates clearly from the active metal atom centres, which does not depend on the size or surface characteristics of the support. Hence, we believe that mechanistic studies based on SACs will be of general significances.

In nature, heme-copper oxidases (HCOs), such as cytochrome c oxidases (Figure 1a) containing a heterobinuclear $\mathrm{Cu}-\mathrm{Fe}$ centre, can efficiently catalyse the four-electron reduction of dioxygen to water with minimal overpotential in the final step of respiration $^{[23]}$. The released chemical energy in this reaction is subsequently stored into adenosine triphosphate (ATP), which is a common energy molecule in most forms of life ${ }^{[24]}$. As essential micronutrients, small amounts of copper and iron ions will have negligible side effects on tissues and organs ${ }^{[25,26]}$.

Therefore, based on the above considerations, we have developed four types of model SACs with or without $\mathrm{Cu}$ or/and $\mathrm{Fe}$ ions being constrained on a carbon matrix: 
(i) hollow $\mathrm{N}$-doped carbon spheres (HNCS); (ii) single-atoms of $\mathrm{Cu}$ constrained on HNCS (C-HNCS); (iii) single-atoms of Fe constrained on HNCS (F-HNCS); and (iv) heterobinuclear $\mathrm{Cu}$-Fe centres constrained on an HNCS (CF-HNCS). In this study, by using these model SACs and employing chromogenic reaction, voltammetric technique, and density functional theory (DFT) calculations, we find that the oxidase-like and electrochemical ORR activities of catalysts are homologous in nature and quantitatively show a good linear relationship with each other. Thus, a novel, facile but general oxidase-like activity assessment methodology based on the voltammetric technique could be well developed for rapidly screening thousands of potential catalysts as oxidase mimics. Furthermore, we demonstrate the synergistic effect of $\mathrm{Cu}-\mathrm{Fe}$ bi-atoms in biomimetic CF-HNCS catalysts and their remarkably high catalytic activities in both organic oxidation and tumour therapy.

\section{Results}

Design, synthesis, and characterization. Figure 1a illustrates the CF-HNCS synthesis procedure. Briefly, 5,10,15,20-tetra(4-(imidazol-1-yl)phenyl)porphyrindine (TIPP), $\mathrm{Cu}$-TIPP, Fe-TIPP, and $\alpha, \alpha^{\prime}$-dibromo-p-xylene were subject to quaternization reactions (Scheme $\mathrm{S} 1$ ) on the surface of the prepared $\mathrm{SiO}_{2}$ nanospheres (Figure $\mathrm{S} 1 \mathrm{a}$ ) to produce a $\mathrm{SiO}_{2} @ \mathrm{Cu} / \mathrm{Fe}-\mathrm{TIPP} / \mathrm{TIPP}$-polymer (Figure $\mathrm{S} 1 b$ ). Consequently, the $\mathrm{SiO}_{2} @ \mathrm{Cu} / \mathrm{Fe}-$ TIPP/TIPP-polymer was pyrolyzed in a hydrogen/argon atmosphere. Moreover, $\mathrm{Cu}$ and/or Fe moieties within the polymer would be carbo-thermally reduced to form isolated single metallic $\mathrm{Cu}, \mathrm{Fe}$ and bimetallic $\mathrm{Cu}-\mathrm{Fe}$ sites coordinated with pyridinic $\mathrm{N}$. 
Finally, the $\mathrm{SiO}_{2}$ core was etched off by $\mathrm{NH}_{4} \mathrm{HF}_{2}$, leading to the CF-HNCS formation. In Figure $1 \mathrm{~b}$ and Figure S1c, CF-HNCS of $\approx 390 \mathrm{~nm}$ in diameter displays a uniform size distribution and maintains spherical $\mathrm{SiO}_{2}$ morphology.

Figure 1c shows an aberration-corrected high-angle annular dark-field scanning transmission electron microscopy (AC HAADF-STEM) image of CF-HNCS. The bright single dots and dual dots confirm the existence of single $\mathrm{Cu}$ atoms, single $\mathrm{Fe}$ atoms and dual $\mathrm{Cu}-\mathrm{Fe}$ atoms because of the heavier $\mathrm{Cu}$ and $\mathrm{Fe}$ atoms than the nitrogendoped carbon support. The high-resolution TEM (HR-TEM) image and fast Fourier transform (FTT) pattern show that a small part of carbon matrix has crystallized, and the graphite layer with a d-spacing of $\sim 0.35 \mathrm{~nm}$ can be clearly seen, which can be attributed to a (002) lattice plane(Figure 1d). ${ }^{[27]}$ The HAADF-STEM results and the corresponding energy-dispersive X-ray spectroscopy (EDS) mapping results demonstrate the uniform distributions of $\mathrm{N}, \mathrm{Cu}$, and $\mathrm{Fe}$ in the carbon matrix (Figure 1ei).

Fine structural characterization. To probe the chemical structures of the $\mathrm{Cu}$ and $\mathrm{Fe}$ atoms in-depth, X-ray absorption spectroscopy (XAS) was adopted. As shown in the $\mathrm{Cu}$ K-edge XANES profiles, the solid red line intensity and the pre-edge peak of CFHNCS and C-HNCS are located in between those of $\mathrm{Cu}_{2} \mathrm{O}$ and $\mathrm{CuO}$, revealing that the valence of copper in the SACs is between +1 and +2 (Figure 2a and Figure S3a). Furthermore, the oxidation state of Fe ions in CF-HNCS and F-HNCS was determined to be between +2 and +3 from the Fe K-edge XANES profiles (Figure $2 \mathrm{~b}$ and Figure $\mathrm{S} 4 \mathrm{a})$. To examine the local coordination environment of $\mathrm{Cu}$ and $\mathrm{Fe}$ sites, we 
investigated the extended X-ray absorption fine structure (EXAFS) of the SACs. Fourier transformed EXAFS (FT-EXAFS) analysis of the Cu K-edge in $R$ space shows that the peaks at $2.22 \AA$ (uncorrected) present in the $\mathrm{Cu}-\mathrm{Cu}$ shell of $\mathrm{Cu}$-foil are absent in $\mathrm{CF}-\mathrm{HNCS}$ and $\mathrm{C}-\mathrm{HNCS}$, implying that most of $\mathrm{Cu}$ atoms are isolated (Figure $2 \mathrm{c}$ and Figure S3b-d). Similarly, the FT-EXAFS spectra of Fe K-edge reveal that Fe atoms are also atomically dispersed in CF-HNCS and F-HNCS (Figure 2d and Figure S4b-d). However, it can be found that $\mathrm{Cu}-\mathrm{Fe}$ bonding probably exists in CF-HNCS from the extracted accurate coordination numbers $(\mathrm{CNs})$ and bond lengths, suggesting that part of the copper species is in the chemical interaction with the Fe species (Figure 2e,f and Table S1,2). The CNs of Cu-Fe bonds in CF-HNCS samples are 0.9 from both $\mathrm{Cu} \mathrm{K}$ edge and Fe K-edge EXAFS fitting curves, confirming that the interacting metal atoms exist mainly in the form of bimetallic $\mathrm{Cu}-\mathrm{Fe}$ atomic clusters in $\mathrm{Cu}-\mathrm{Fe}-\mathrm{N}-\mathrm{C}$ rather than in the form of metal-based nanoparticles. The $\mathrm{Cu}-\mathrm{Fe}$ bond length is approximately $3 \AA$ in CF-HNCS, which is close to the d-spacing of the graphite layer (3.5 $⿱$ ) $)$ in the matrix. The CNs of $\mathrm{Cu}-\mathrm{N}$ and $\mathrm{Fe}-\mathrm{N}$ bonds in the first shell are similarly and approximately 4, implying the similar $\mathrm{Cu}-\mathrm{N}_{4}$ and $\mathrm{Fe}-\mathrm{N}_{4}$ bonding forms in the $\mathrm{N}$-doped carbon matrix. In summary, each $\mathrm{Cu}$ or $\mathrm{Fe}$ atom is coordinated with four $\mathrm{N}$ atoms to form $\mathrm{Cu}-\mathrm{N}_{4}$ or $\mathrm{Fe}-$ $\mathrm{N}_{4}$ sites in C-HNCS or F-HNCS, respectively (Figure S3f, 4f); While most metal atoms are isolated in the form of metal-N 4 , and a small part of the metal species are anchored on neighbouring graphite layers to form $\mathrm{Cu}-\mathrm{Fe}$ dual atomic sites in $\mathrm{CF}-\mathrm{HNCS}-1$ (Figure $2 \mathrm{~g}$ ). Because the $\mathrm{Cu}$ and $\mathrm{Fe}$ atoms anchored on the same carbon layer may also have synergetic interactions, we propose an architecture of $\mathrm{Fe}-\mathrm{Cu}$ dual atomic sites in $\mathrm{CF}$ - 
HNCS-2 (Figure 2h), though this long-range interaction is hard to detect.

Furthermore, wavelet transform (WT) analysis was performed to verify the backscattering atom. As illustrated in Figure $2 \mathrm{i}, \mathrm{k}$, the first intensity maximum of $\mathrm{Cu}$ Fe-N-C at $1.40 \AA$ (uncorrected) correlated with a k-value of $\sim 4.2 \AA^{-1}$, and that at 1.45 $\AA$ (uncorrected) correlated with a k-value of $\sim 4.9 \AA^{-1}$, could be ascribed to $\mathrm{Cu}-\mathrm{N}$ bonding and Fe-N bonding, respectively. Compared with the WT plots of $\mathrm{Cu}$ and $\mathrm{Fe}$ foils (Figure 2i,k), the intensity maxima corresponding to $\mathrm{Cu}-\mathrm{Cu}$ and $\mathrm{Fe}-\mathrm{Fe}$ are absent, further confirming that there are no metal-based nanoparticles. The second intensity maximum of Cu-Fe-N-C at $2.66 \AA$ (uncorrected) correlated with a k-value of $\sim 7.0 \AA^{-1}$ (Figure $2 \mathrm{j}$ ), and that at $2.75 \AA$ (uncorrected) correlated with a k-value of $\sim 7.9 \AA^{-1}$ (Figure 21), could be attributed to $\mathrm{Cu}-\mathrm{Fe}$ bonding. Though the second intensity maximum could also be speculated as $\mathrm{Cu}-\mathrm{Cu}$ or Fe-Fe bonding, such a possibility could be ignored due to the substantially higher catalytic activity of the heteronuclear $\mathrm{Cu}-\mathrm{Fe}$ dual-atomic catalyst in previous reports and the formation of an ideal structure in natural human cytochrome c oxidase (PDB 5Z62) ${ }^{[23,28]}$. The WT analysis demonstrates that $\mathrm{Cu}-\mathrm{Fe}-\mathrm{N}-\mathrm{C}$ truly contains dual-atomic sites, consistent with the EXAFS fitting parameters. These structural characterizations show that the $\mathrm{CF}-\mathrm{HNCS}$ has $\mathrm{Cu}-\mathrm{Fe}$ active sites similar to those of HCOs.

Catalytic performance. The synergistic catalytic effect between copper and iron atoms in the CF-HNCS catalysts may endow the catalysts with excellent oxidase-like performance. To investigate the oxidase-like activities of the model catalysts, we 
employed the oxidation of 3,3',5,5'-tetramethylbenzidine (TMB) as a model chromogenic reaction. In deionized water, the velocity of the chromogenic reaction (Figure 3a) shows the oxidase-like activity order of CF-HNCS $>$ C-HNCS + F-HNCS $>$ F-HNCS $>$ C-HNCS $\gg$ HNCS, indicating that the synergistic effect and the active metal atom are two important factors for the catalytic activity.

In fuel cells, the cathode receives oxidants (often oxygen), and the anode receives reductants, such as some organics. ${ }^{[29,30]}$ The ORR is the half-reaction in the oxidization of reductant by $\mathrm{O}_{2}$. From a chemical reaction viewpoint, if the ORR activity of the catalyst is elevated, its capacity for catalysing organic oxidation by $\mathrm{O}_{2}$ would be enhanced under the same reaction conditions, especially when the rate-determining steps (RDSs) are in the processes of desorption and adsorption of $\mathrm{O}_{2}$. Considering the remarkable ORR activity of commercial $\mathrm{Pt} / \mathrm{C}, \mathrm{Pt} / \mathrm{C}$ may exhibit excellent activity in catalysing $\mathrm{O}_{2}$ reduction to oxidize the organics. The velocity of the chromogenic reaction in deionized water (Figure 3a) and the time-dependent absorbance of TMB in NaAc buffer solution (Figure S5a) show that the oxidase-like activity of commercial $\mathrm{Pt} / \mathrm{C}$ is significantly higher than that of CF-HNCS. Nevertheless and notably, the calculated turnover frequency of $\mathrm{Cu}-\mathrm{Fe}$ in $\mathrm{CF}-\mathrm{HNCS}$ was approximately 0.93 times that of $\mathrm{Pt}$ in $\mathrm{Pt} / \mathrm{C}$, revealing the outstanding oxidase-like activity of CF-HNCS. Then, we investigated the ORR activity of all samples by steady-state linear sweep voltammetry (LSV) in various solutions at room temperature $\left(\sim 25^{\circ} \mathrm{C}\right)$. The obtained order of ORR activity is: Pt/C $>$ CF-HNCS $>$ F-HNCS $>$ C-HNCS $\gg$ HNCS in both $\mathrm{O}_{2}$-saturated deionized water and $\mathrm{O}_{2}$-saturated $\mathrm{NaAc}$ buffer solution (Figure $3 \mathrm{~b}$ and 
Figure S5b), which are in excellent agreement with that of oxidase-like activity identified by TMB (Figure 3a and Figure S5c), suggesting that the oxidase-like and ORR activity may follow the same mechanism and possess the same active sites. The nondimensionalized velocity $\left(v_{\mathrm{n}}\right)$ of the chromogenic reaction at varied TMB concentrations and the absolute value of the normalized mean current density $\left(\left|\overline{j_{\mathrm{n}}}\right|\right)$ from 1 to $0 \mathrm{~V}$ in ORR are used for representing the oxidase-like and ORR activities of various catalysts, respectively. Figure $3 \mathrm{~b}$ and f show the excellent fitting linearity between $v_{\mathrm{n}}$ and $\left|\overline{j_{n}}\right|$ in both deionized water and NaAc buffer solution, in which all Pearson correlation coefficients (Person's r) are larger than 0.985. The slopes and intercepts increase with the increase of TMB concentration from 0.75 to $0.25 \mathrm{mM}$, indicating that the enzyme becomes saturated when the substrate concentration increases. Especially, the linear relation at 0.25 and $0.125 \mathrm{mM}$ are almost the same with each other, demonstrating that $\mathrm{y}_{0.125}$ and $\mathrm{y}_{0.25}$ could well-describe the relation between oxidase-like and ORR activity in the linear part of enzyme reactions. Thus, the $\left|\overline{j_{\mathrm{n}}}\right|$ is an effective descriptor for the catalytic activity of the oxidase mimics. Based on the above analysis, we propose that the oxygen reduction electrocatalysts possess homologous oxidase-like activity by sharing the same activity origins, so that the conventional LSV technique is readily applicable to describe the oxidase-like activity. Furthermore, the ORR activity also follows the order of $\mathrm{Pt} / \mathrm{C}>\mathrm{CF}-\mathrm{HNCS}>\mathrm{F}-\mathrm{HNCS}>\mathrm{C}-\mathrm{HNCS} \gg \mathrm{HNCS}$ in an $\mathrm{O}_{2}-$ saturated $0.1 \mathrm{M} \mathrm{HClO}_{4}$ solution (Figure 3d), implying that a large number of reported oxygen reduction electrocatalysts with remarkable activities might be promising candidates for oxidase mimics. 
In addition, the currently popular electron spin resonance (ESR), fluorescence and chromogenic methodologies for assessing oxidase-like activity are difficult to be applied in high-glucose Dulbecco's modified Eagle medium (DMEM), which is used in cell culture but complicated in composition. However, the LSV technique works well in DMEM. The ORR activity order in $\mathrm{O}_{2}$-saturated SBF and $\mathrm{O}_{2}$-saturated DMEM solutions is: CF-HNCS $>\mathrm{Pt} / \mathrm{C}>\mathrm{F}-\mathrm{HNCS}>\mathrm{C}$-HNCS $\gg$ HNCS (Figure 3e,f). Interestingly, the ORR activity of CF-HNCS here is higher than that of $\mathrm{Pt} / \mathrm{C}$ in DMEM and SBF solutions, indicating that glucose and anions such as chloride and phosphate anions may poison $\mathrm{Pt} / \mathrm{C}^{[31,32]}$ and $\mathrm{CF}-\mathrm{HNCS}$ could be a better cancer therapeutic catalyst owing to the great anti-poisoning effect and the high-performance catalytic activity. The cyclic voltammograms (CVs) at the CF-HNCS electrodes were obtained to reveal the influence of solutions on the electrochemical performance. As shown in Figure S5d, the numbers of oxidation and reduction peaks were different in different solutions due to the changed solute, revealing that the DMEM solution offers the maximum number of redox couples and that these redox reactions can be catalysed by CF-HNCS. Based on this, the CV technique could be well-used to identify the redox couples catalysed by oxidase mimics in different solutions.

From an electrochemical viewpoint, reactive oxygen species (ROS) are generated as intermediates during the ORR, ${ }^{[33]}$ which is consistent with the pathway of oxidasebased enzymatic catalysis. ${ }^{[13,34-36]}$ Therefore, we employed ESR to monitor the possible active intermediate production. The six-band spectra are originated from $\mathrm{BMPO} / \cdot \mathrm{OOH}$, and the band intensity follows the order of CF-HNCS $>$ F-HNCS $>$ C-HNCS $\gg$ HNCS 
(Figure S5e), demonstrating that the ROS generation performance corresponds well to the ORR catalytic activity characterized by LSV and the oxidase-like activity characterized by TMB. In addition, significant fluorescence enhancement of dihydroethidium (DHE) was observed in the presence of CF-HNCS, demonstrating the generation of $\mathrm{O}_{2} \bullet^{-}$(Figure S5f). Glutathione (GSH) is a reducing species in tumour cell redox homeostasis, ${ }^{[37]}$ and the fluorescence intensity reaches its maximum by adding both CF-HNCS and GSH, suggesting that GSH can act as a promoter in the oxidation of DHE. This result also implies that GSH can be oxidized and may promote CF-HNCS catalysis towards the oxidation of biomolecules in cells.

DFT calculations. DFT calculations were performed to investigate the mechanism and the shared origins of the oxidase-like/ORR activity. We hypothesize a $4 \mathrm{e}^{-}$reduction pathway in which an $\mathrm{O}_{2}$ molecule will be first adsorbed and protonated to form $* \mathrm{OOH}$ on top of central $\mathrm{Cu}$ or $\mathrm{Fe}$ atoms. Then, the ${ }^{*} \mathrm{OOH}$ will associate with $\mathrm{H}^{+}$and dissociate into*O and $\mathrm{H}_{2} \mathrm{O}$. Next, the $* \mathrm{O}$ will be protonated to form $* \mathrm{OH}$. Finally, ${ }^{*} \mathrm{OH}$ will associate with $\mathrm{H}^{+}$to generate the final product $\mathrm{H}_{2} \mathrm{O}$ (Figure 4a and Figure $\mathrm{S} 6$ ).

The free energy profiles in Figure $4 \mathrm{~b}$ show that the ORR overpotentials for CHNCS, CF-HNCS-1-Cu (CF-1-Cu), CF-HNCS-2-Cu (CF-2-Cu), F-HNCS, CF-HNCS1-Fe (CF-1-Fe) and CF-HNCS-2-Fe (CF-2-Fe) were estimated to be $0.96 \mathrm{~V}, 0.77 \mathrm{~V}$, $0.82 \mathrm{~V}, 0.80 \mathrm{~V}, 0.59 \mathrm{~V}$, and $0.81 \mathrm{~V}$, respectively. Based on the free energy values, the calculated order of ORR activity is CF-HNCS-1 $>$ CF-HNCS-2 $>$ F-HNCS $>$ C-HNCS, in accordance with the experimental findings. Figure $4 \mathrm{~b}$ shows that the first step is the 
rate-determining step (RDS) on $\mathrm{Cu}$, while the fourth step is the RDS on Fe. Therefore, the superior ORR activity of dual atomic CF-HNCS-1 to the other single atomic catalysts is ascribed to the enhanced adsorption of ${ }^{*} \mathrm{OOH}$ at the $\mathrm{Cu}$ site, which facilitates the protonation of $\mathrm{O}_{2}$ on $\mathrm{CF}-1-\mathrm{Cu}$, and the weakened adsorption of $* \mathrm{OH}$ at the Fe site, which facilitates the removal of $\mathrm{H}_{2} \mathrm{O}$ from CF-1-Fe. In CF-HNCS-2, the overpotential of CF-2-Fe increases slightly compared to the Fe site in F-HNCS, while that of CF-2-Cu decreases significantly to $0.77 \mathrm{~V}$, which is even lower than that of the Fe sites in F-HNCS and CF-HNCS-2. Therefore, the dual $\mathrm{Cu}$ and Fe atomic system of the lowest overpotential demonstrates the highest ORR activity in comparison to the corresponding single atomic $\mathrm{Cu}$ or Fe systems.

Figure $4 c$ shows that the thermodynamic relation between the ORR overpotentials and the $\mathrm{O}_{2}$ binding energies roughly form a volcano-type curve. On the left side, the fourth step $\left({ }^{*} \mathrm{OH}+\mathrm{H}^{+}+\mathrm{e}^{-} \rightarrow \mathrm{H}_{2} \mathrm{O}\right)$ is the RDS, suggesting that the over-high $\mathrm{O}_{2}$ binding energy makes the adsorption and dissociation of * $\mathrm{OH}$ species less favourable. In contrast, the first step $\left(\mathrm{O}_{2}+\mathrm{H}^{+}+\mathrm{e}^{-} \rightarrow{ }^{*} \mathrm{OOH}\right)$ is the $\mathrm{RDS}$ on the right side, revealing that the over-weak adsorption and activation of $\mathrm{O}_{2}$ molecules will increase the difficulty in the protonation of $\mathrm{O}_{2}$. CF-1-Fe has an appropriate $\mathrm{O}_{2}$ binding energy, endowing the catalyst with the optimal ORR activity. These results imply that optimizing the binding strength of $\mathrm{O}_{2}$ on catalytic sites is an efficient way to achieve excellent ORR activity. Finally, the free energy profiles of the ORR under different $\mathrm{pH}$ values $(1.0,4.5,6.0,7.4)$ are given in Figure S7. The most energetically favourable $\mathrm{pH}$ value is 1.0 , implying that a reasonably strong acidic environment is beneficial for 
improving the catalytic performance. Figure S7d shows that the reaction process has a negative Gibbs free energy $(-3.17 \mathrm{eV})$ and a surmountable apparent energy barrier $(0.17$ $\mathrm{eV}$ ) even at $\mathrm{pH}=$ 7.4. Accordingly, the DFT results indicate that it is both thermodynamically feasible and kinetically favourable for the ORR to be effectively catalysed at the active sites from $\mathrm{pH}=0$ to 7.4 .

We have probed the catalytic mechanism and the shared origins of the oxidaselike/ORR activities of the model single-atom catalysts at the atomic level, and identified that the metal active sites and the $\mathrm{Cu}-\mathrm{Fe}$ synergistic effect are two key factors for the catalytic activity. In addition, CF-HNCS-1 has the most favourable catalytic structure, indicating that biomimetic synthesis is an effective way to develop next-generation oxidase-like/ORR catalysts.

In vitro catalytic therapeutic performance. Encouraged by the excellent oxidase-like activity of CF-HNCS catalysts for the generation of ROS and the oxidation of organic molecules such as GSH, we further explored the in vitro anticancer efficacy. First, the Cell Counting Kit-8 (CCK-8) assay was used to investigate the catalytic therapeutic efficacy against 4T1 tumour cells. As expected, a dose-dependent cellular killing effect was observed, as shown in Figure 5a. Moreover, the reduction rates in 4T1 tumour cell viability reached $58 \%$ and $69 \%$ at CF-HNCS concentrations of 50 and $200 \mathrm{ppm}$, respectively, in which the metallic $\mathrm{Cu}$ and Fe concentrations were as low as 0.93 and $3.72 \mathrm{ppm}$, respectively. More interestingly, the cellular killing effect also follow the order of CF-HNCS $>$ F-HNCS $>$ C-HNCS $>$ HNCS, implying that the oxidase-like 
activities of the catalysts are responsible for the significant anticancer effects. Due to the relatively low redox potential of $\mathrm{O}_{2} \bullet^{-}$and the complicated cell environment, we have been not able to detect the oxidase-like activities of the catalysts directly in the cell. Furthermore, flow cytometric analysis was conducted to study the apoptosis of 4T1 cancer cells by staining with annexin V-FITC and PI. Necrotic ( $\left.Q_{1}\right)$, late apoptotic $\left(\mathrm{Q}_{2}\right)$, early apoptotic $\left(\mathrm{Q}_{3}\right)$ and live $\left(\mathrm{Q}_{4}\right)$ cells are presented in the four quadrants in Figure 5b. A similar trend of the cell apoptosis order was also obtained. The sum of early and late cellular apoptotic rates is up to $76.0 \%$ in the CF-HNCS group, showing the prominent therapeutic efficacy in vitro.

In vivo catalytic therapeutic performance. Compared to conventional injection methods, the microneedle (MN) patch offers a painless and minimally invasive selfadministration route, avoiding the risk of bleeding and injury. ${ }^{[38,39]}$ In this study, we fabricated a polyvinyl pyrrolidone (PVP)-based MN patch for the delivery of CFHNCS catalysts (Figure S8a-e). First, different doses of CF-HNCS catalysts were confirmed to have excellent biocompatibility in healthy Kunming mice (Figure S9a-c). A more detailed analysis of the biocompatibility experiment is given in the above discussion. Furthermore, the therapeutic efficacies of these synthesized catalysts for catalytic tumour therapy was assessed in 4T1 xenografted tumour-bearing mice. The CF-HNCS-MN was intratumourally injected, and then the MN dissociated itself and released catalysts for cancer therapy. There were slight fluctuations in the weights of these tumour-bearing mice, demonstrating the good biocompatibility of the $\mathrm{MN}$ 
(Figure 6a). Importantly, CF-HNCS, F-HNCS and C-HNCS catalysts show remarkable effects in suppressing tumour growth (Figure 6b). The relative tumour inhibition (RTI) rates compared to the control group were calculated to quantify the therapeutic performance. The RTI rates of the experimental groups treated with catalysts gradually increase and reach their maxima at the end of the observations, indicating the effective long-term suppression of tumour growth by the catalysts (Figure 6c). In particular, the RTI rate reaches $94 \%$ on day 14 in the CF-HNCS group. In addition, we have found that the overall survival length in the CF-HNCS group is more than twice that in the control group, showing a significant elevation in survival rates after catalyst treatment (Figure 6d). The therapeutic performance was also evaluated by histological analysis using pathological haematoxylin and eosin (H\&E) (Figure 6e), Ki-67 antibody (Figure 6f), and terminal deoxynucleotidyl transferase dUTP nick end labeling (TUNEL) staining (Figure 6g). After catalytic therapy, the tumour tissues exhibit prominent cell fibrosis, apoptosis and necrosis (Figure 6e), significantly suppressed proliferation (Figure 6f), serious cell apoptosis and fragmentation of DNA (Figure 6g). Most importantly, the catalytic therapeutic outcome in vivo also follows the order of CFHNCS $>$ F-HNCS $>$ C-HNCS $>$ HNCS, confirming that the oxidase-like/ORR activity of materials is responsible for the inhibition of tumours. These results demonstrate that we could search and screen effective therapeutic nanocatalysts by assessing their oxidase-like, i.e., ORR activity, before in vivo experiments, which is expected to accelerate the screening of potential therapeutic nanocatalysts.

\section{Discussion.}


In summary, we present a proof-of-concept study of bridging the biological oxidase-like and electrochemical ORR catalysis, both experimentally and theoretically, by using model single atom catalysts. Importantly, the oxidase-like and electrochemical ORR catalytic activity have been demonstrated to be linearly co-related with each other based on their shared identical activity origins, thus the electrocatalytic characterizations, such as LSV curves, could be the reliable, facile and efficient ways to be used for searching oxidase-mimics. Quantitatively, the normalized mean current density $\left|\overline{j_{\mathrm{n}}}\right|$ of cathodic ORR has been found to be an apparent descriptor for characterizing the oxidase-like activity of the catalyst. Resultantly, great numbers of high-activity oxygen reduction electrocatalysts are expected to possess prominent oxidase-like activity, which encourages us to find more types of electrochemical catalysts and then verify their enzyme-like catalytic activity in future. Furthermore, both experimental and theoretical calculations demonstrate that the biomimetic heterobinuclear $\mathrm{Cu}-\mathrm{Fe}$ centres show well-balanced, i.e., optimal adsorption strength of oxygenated species on the active sites and the significant synergistic effect between the two kinds of metal atoms, which is responsible for the outstanding catalytic activities. Our findings suggest the promising prospects of designing high-performance biomimetic enzymes via facilely designing and synthesizing desirable metal centres as the active sites for ORR, and pave the way for the electrocatalyst's biomedical and environmental applications in, such as tumour therapy, antibiosis, and oxidation degradation of organic pollutants. 


\section{Author contributions}

X.L. and J.S. conceived and designed experiments. X.L. performed most of the experiments. S.G. took part in the cell and animal experiments. H.L. assisted with the animal experiments. H.T. helped with the electrochemical measurements. D.X. assisted with the catalyst synthesis. X.L. and J.S. analyzed the experimental data and wrote the paper. All the authors discussed the results and commented on the manuscript.

\section{Acknowledgements}

Animal experiment procedures were approved and followed the guidelines for the Animal Care Ethics Commission of Shanghai Tenth People's Hospital, Tongji University School of Medicine. We thank to Chen Zhang from Shanghai Institute of Ceramics, Chinese Academy of Sciences for his discussions. The authors thank beamline BL14W1 (Shanghai Synchrotron Radiation Facility) for providing the beam time.

\section{Funding}

This work was supported by the financial support from National Natural Science Foundation of China (grant no. 21835007) and Shanghai Municipal Government S\&T Project (Grant No.17JC1404701).

\section{Competing financial interests}

The authors declare no competing financial interests. 


\section{References}

[1] Liang M, Yan X. Nanozymes: From New Concepts, Mechanisms, and Standards to Applications. Acc Chem Res [J]. 2019, 52: 2190-2200. 10.1021/acs.accounts.9b00140.

[2] Manea F, Houillon F B, Pasquato L, et al. Nanozymes: Gold-Nanoparticle-Based Transphosphorylation Catalysts. Angew Chem Int Ed [J]. 2004, 43: 6165-6169. 10.1002/anie.200460649.

[3] Gao L, Zhuang J, Nie L, et al. Intrinsic peroxidase-like activity of ferromagnetic nanoparticles. Nat Nanotechnol [J]. 2007, 2: 577-583. 10.1038/nnano.2007.260.

[4] Jiang D, Ni D, Rosenkrans Z T, et al. Nanozyme: new horizons for responsive biomedical applications. Chem Soc Rev [J]. 2019, 48: 3683-3704. 10.1039/c8cs00718g.

[5] He F, Mi L, Shen Y, et al. Fe-N-C Artificial Enzyme: Activation of Oxygen for Dehydrogenation and Monoxygenation of Organic Substrates under Mild Condition and Cancer Therapeutic Application. ACS Appl Mater Interfaces [J]. 2018, 10: 35327-35333. 10.1021/acsami.8b15540.

[6] Dong K, Lei Y, Zhao H, et al. Noble-metal-free electrocatalysts toward $\mathrm{H} 2 \mathrm{O} 2$ production. $J$ Mater Chem A [J]. 2020, 8: 23123-23141. 10.1039/D0TA08894C.

[7] Liang J, Wang Y, Liu Q, et al. Electrocatalytic hydrogen peroxide production in acidic media enabled by NiS2 nanosheets. J Mater Chem A [J]. 2021, 9: 6117-6122. 10.1039/D0TA12008A.

[8] Lu X, Gao S, Lin H, et al. Bioinspired Copper Single-Atom Catalysts for Tumor Parallel Catalytic Therapy. Adv Mater [J]. n/a: 2002246. 10.1002/adma.202002246.

[9] Huang L, Chen J, Gan L, et al. Single-atom nanozymes. Science Sci Adv [J]. 2019, 5: eaav5490. 10.1126/sciadv.aav5490.

[10] Wang L, Hu C, Shao L. The antimicrobial activity of nanoparticles: present situation and prospects for the future. Int J Nanomedicine [J]. 2017, 12: 1227-1249. 10.2147/IJN.S121956.

[11] Yang B, Chen Y, Shi J J C R. Reactive oxygen species (ROS)-based nanomedicine. Chem Rev [J]. 2019, 119: 4881-4985. 10.1021/acs.chemrev.8b00626.

[12] Singh S. Nanomaterials Exhibiting Enzyme-Like Properties (Nanozymes): Current Advances and Future Perspectives. Front Chem [J]. 2019, 7: 46. 10.3389/fchem.2019.00046.

[13] Fan K, Xi J, Fan L, et al. In vivo guiding nitrogen-doped carbon nanozyme for tumor catalytic therapy. Nat Commun [J]. 2018, 9: 1440. 10.1038/s41467-018-03903-8.

[14] Mano N, De Poulpiquet A. O2 Reduction in Enzymatic Biofuel Cells. Chemical Reviews [J]. 2018, 118: 2392-2468. 10.1021/acs.chemrev.7b00220.

[15] Mano N, Mao F, Heller A. A Miniature Biofuel Cell Operating in A Physiological Buffer. J Am Chem Soc [J]. 2002, 124: 12962-12963. 10.1021/ja028514g.

[16] Climent V, Fu Y, Chumillas S, et al. Probing the Electrocatalytic Oxygen Reduction Reaction Reactivity of Immobilized Multicopper Oxidase CueO. J Phys Chem C [J]. 2014, 118: 1575415765. 10.1021/jp5034382.

[17] Guo D, Shibuya R, Akiba C, et al. Active sites of nitrogen-doped carbon materials for oxygen reduction reaction clarified using model catalysts. Science [J]. 2016, 351: 361-365. 10.1126/science.aad0832.

[18] Liu M, Wang L, Zhao K, et al. Atomically dispersed metal catalysts for the oxygen reduction reaction: synthesis, characterization, reaction mechanisms and electrochemical energy applications. Energy Environ Sci [J]. 2019, 12: 2890-2923. 10.1039/C9EE01722D.

[19] Xiangyu Lu S G, Han Lin, and Jianlin Shi. Single-Atom Catalysts for Nanocatalytic Tumor 
Therapy. Small [J]. 2020. 10.1002/smll.202004467.

[20] Wang A, Li J, Zhang T. Heterogeneous single-atom catalysis. Nature Reviews Chemistry [J]. 2018, 2: 65-81. 10.1038/s41570-018-0010-1.

[21] Cao L, Luo Q, Liu W, et al. Identification of single-atom active sites in carbon-based cobalt catalysts during electrocatalytic hydrogen evolution. Nat Catal [J]. 2019, 2: 134-141. 10.1038/s41929-018-0203-5.

[22] Cui X, Li W, Ryabchuk P, et al. Bridging homogeneous and heterogeneous catalysis by heterogeneous single-metal-site catalysts. Nat Catal [J]. 2018, 1: 385-397. 10.1038/s41929018-0090-9.

[23] Zong S, Wu M, Gu J, et al. Structure of the intact 14-subunit human cytochrome c oxidase. Cell Res [J]. 2018, 28: 1026-1034. 10.1038/s41422-018-0071-1.

[24] Halime Z, Kotani H, Li Y, et al. Homogeneous catalytic $\mathrm{O}<$ sub $>2</$ sub $>$ reduction to water by a cytochrome $<\mathrm{em}>\mathrm{c}</ \mathrm{em}>$ oxidase model with trapping of intermediates and mechanistic insights. Proc Natl Acad Sci U S A [J]. 2011, 108: 13990-13994. 10.1073/pnas.1104698108.

[25] Burkhead J L, Gogolin Reynolds K A, Abdel-Ghany S E, et al. Copper homeostasis. New Phytol [J]. 2009, 182: 799-816. 10.1111/j.1469-8137.2009.02846.x.

[26] Gozzelino R, Arosio P. Iron Homeostasis in Health and Disease. Int J Mol Sci [J]. 2016, 17. 10.3390/ijms17010130.

[27] Sheng Z-H, Shao L, Chen J-J, et al. Catalyst-Free Synthesis of Nitrogen-Doped Graphene via Thermal Annealing Graphite Oxide with Melamine and Its Excellent Electrocatalysis. ACS Nano [J]. 2011, 5: 4350-4358. 10.1021/nn103584t.

[28] Li F, Liu X, Chen Z. 1 + 1'> 2: Heteronuclear Biatom Catalyst Outperforms Its Homonuclear Counterparts for CO Oxidation. Small [J]. 2019, 3: 1800480. 10.1002/smtd.201800480.

[29] Saikia K, Kakati B K, Boro B, et al. Current Advances and Applications of Fuel Cell Technologies[C]/P. K. SARANGI, S. NANDA, P. MOHANTY. Recent Advancements in Biofuels and Bioenergy Utilization. Singapore:Springer Singapore,2018:303-337. 10.1007/978-981-13-1307-3_13.

[30] Cracknell J A, Vincent K A, Armstrong F A. Enzymes as Working or Inspirational Electrocatalysts for Fuel Cells and Electrolysis. Chem Rev [J]. 2008, 108: 2439-2461. 10.1021/cr0680639.

[31] Arruda T M, Shyam B, Ziegelbauer J M, et al. Investigation into the Competitive and SiteSpecific Nature of Anion Adsorption on Pt Using In Situ X-ray Absorption Spectroscopy. $J$ Phys Chem C [J]. 2008, 112: 18087-18097. 10.1021/jp8067359.

[32] He Q, Shyam B, Nishijima M, et al. Mitigating Phosphate Anion Poisoning of Cathodic Pt/C Catalysts in Phosphoric Acid Fuel Cells. J Phys Chem C [J]. 2013, 117: 4877-4887. 10.1021/jp309282n.

[33] Wang Y, Balbuena P B. Potential Energy Surface Profile of the Oxygen Reduction Reaction on a Pt Cluster: Adsorption and Decomposition of $\mathrm{OOH}$ and $\mathrm{H} 2 \mathrm{O} 2 . J$ Chem Theory Comput [J]. 2005, 1: 935-943. 10.1021/ct0500794.

[34] Wang Z, Zhang Y, Ju E, et al. Biomimetic nanoflowers by self-assembly of nanozymes to induce intracellular oxidative damage against hypoxic tumors. Nat Commun [J]. 2018, 9. 10.1038/s41467-018-05798-x.

[35] Huang X, Groves J T. Oxygen Activation and Radical Transformations in Heme Proteins and Metalloporphyrins. Chem Rev [J]. 2018, 118: 2491-2553. 10.1021/acs.chemrev.7b00373. 
[36] Shen X, Liu W, Gao X, et al. Mechanisms of Oxidase and Superoxide Dismutation-like Activities of Gold, Silver, Platinum, and Palladium, and Their Alloys: A General Way to the Activation of Molecular Oxygen. $J$ Am Chem Soc [J]. 2015, 137: 15882-15891. 10.1021/jacs.5b10346.

[37] Gong N, Ma X, Ye X, et al. Carbon-dot-supported atomically dispersed gold as a mitochondrial oxidative stress amplifier for cancer treatment. Nat Nanotech [J]. 2019, 14: 379-387. 10.1038/s41565-019-0373-6.

[38] Brambilla D, Proulx S T, Marschalkova P, et al. Microneedles for the Noninvasive Structural and Functional Assessment of Dermal Lymphatic Vessels. Small [J]. 2016, 12: 1053-1061. 10.1002/smll.201503093.

[39] Pei P, Yang F, Liu J, et al. Composite-dissolving microneedle patches for chemotherapy and photothermal therapy in superficial tumor treatment. Biomater Sci [J]. 2018, 6: 1414-1423. 10.1039/C8BM00005K. 
a
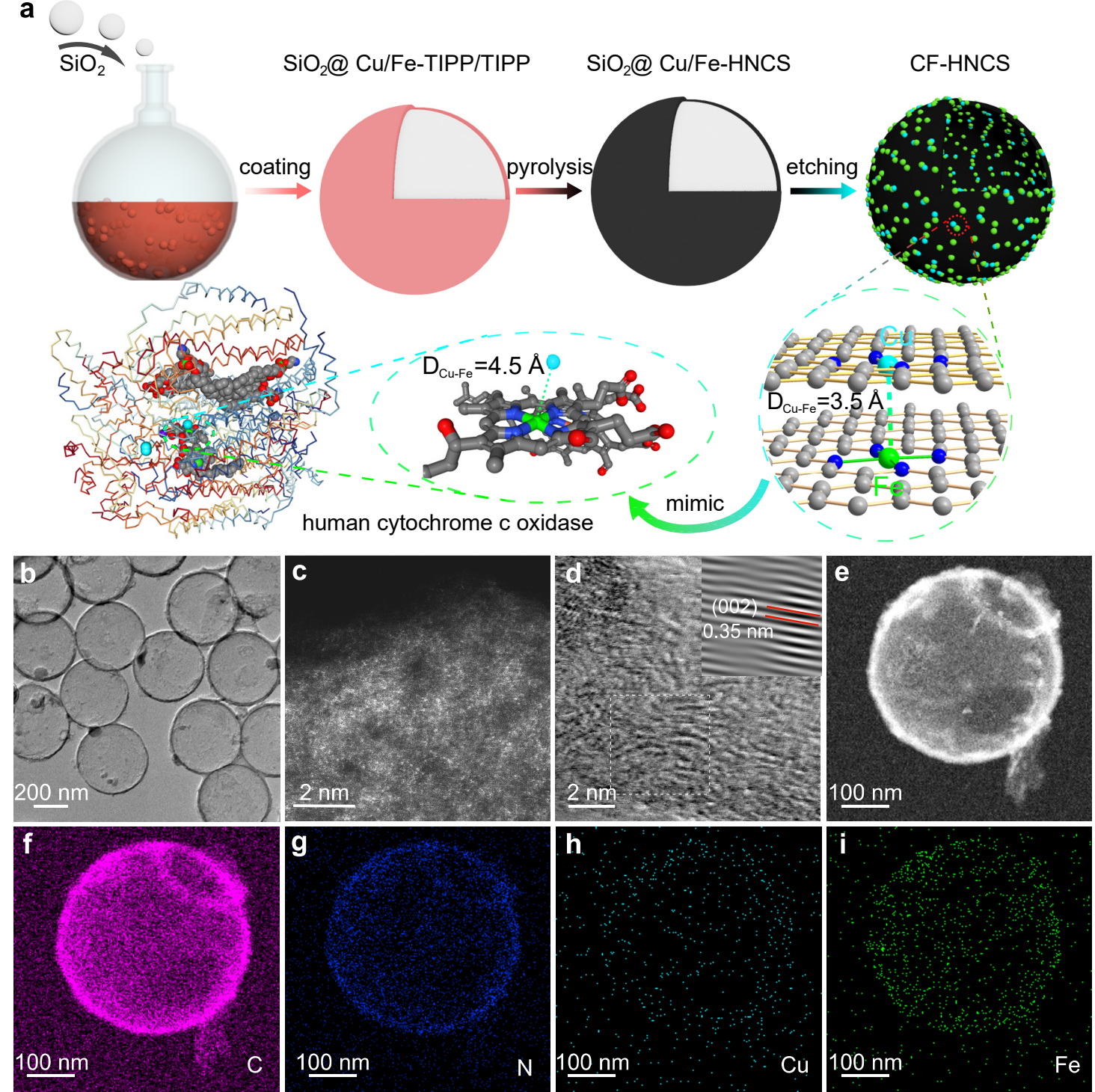

Figure 1. CF-HNCS synthesis and morphological characterization. a, The procedure to synthesize CF-HNCS. b-d, TEM (b), AC HAADF-STEM (c), HR-TEM and the corresponding FTT pattern (inset) of the marked area (d) images of CF-HNCS. e-i, HAADF-STEM image (e) and the corresponding EDS mappings of CF-HNCS: C (f), $\mathrm{N}(\mathbf{g}), \mathrm{Cu}(\mathbf{h})$ and $\mathrm{Fe}(\mathbf{i})$. 

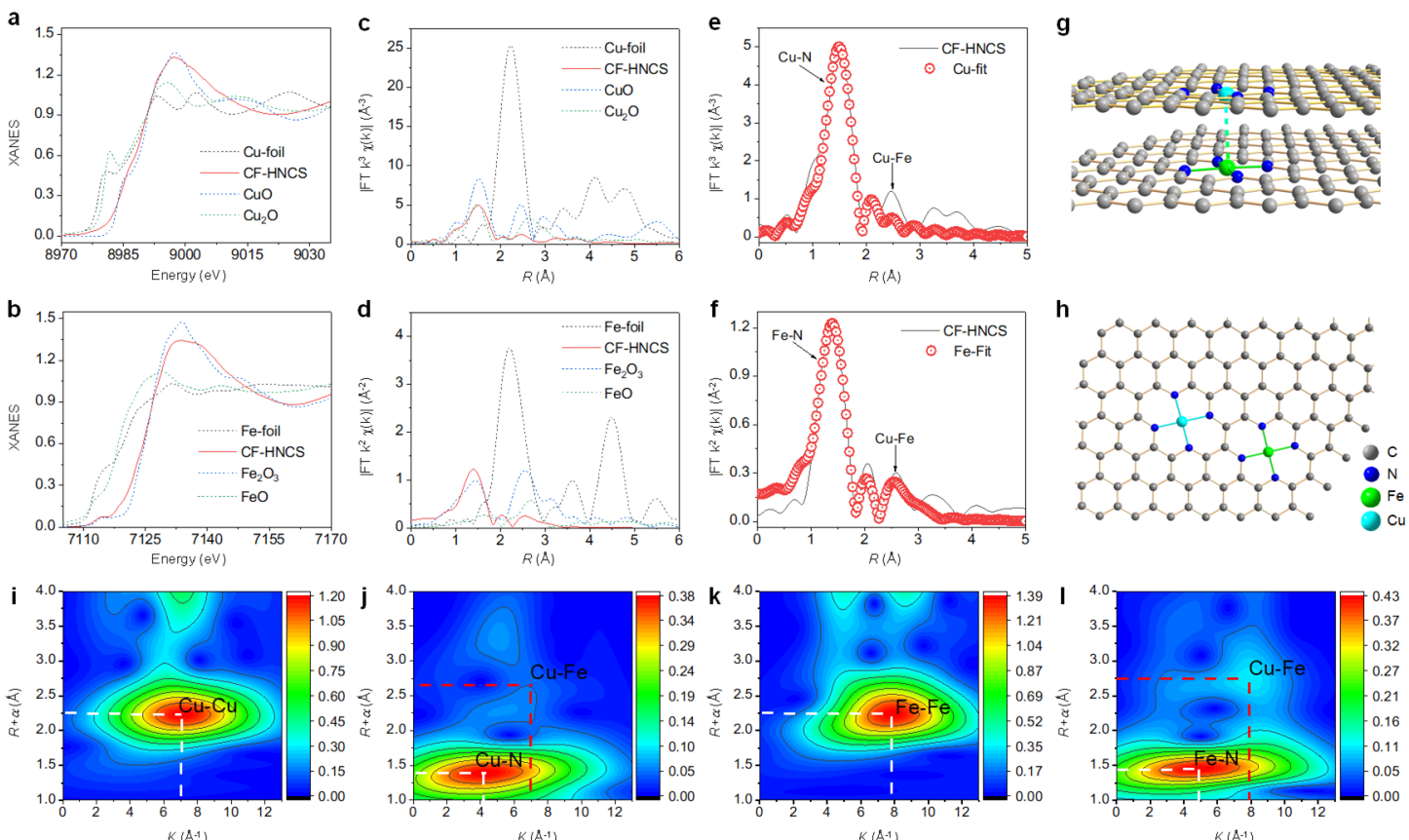

Figure 2. Fine structure characterizations of the active sites. a-b, XANES spectra at the $\mathrm{Cu}$ Kedge (a) and Fe K-edge (b) of CF-HNCS. c-d, Fourier transforms at the $\mathrm{Cu}$ K-edge (c) and Fe Kedge (d) of CF-HNCS. e-f, The corresponding $\mathrm{Cu}$ K-edge (e) and Fe K-edge (f) EXAFS fitting results of $\mathrm{CF}-\mathrm{HNCS}$ in $\mathrm{R}$ space. $\mathbf{g}-\mathbf{h}$, Proposed architecture of $\mathrm{Cu}-\mathrm{Fe}$ dual sites formed on neighbouring but opposite graphite layers (CF-HNCS-1, g) and on the same carbon layer (CFHNCS-2, h). i-j, Wavelet transforms of the $\mathrm{k}^{2}$-weighted $\mathrm{Cu}$ K-edge EXAFS signals of Cu foil (i) and CF-HNCS (j). k-l, Wavelet transforms of the $\mathrm{k}^{2}$-weighted Fe K-edge EXAFS signals of Fe foil (k) and CF-HNCS (l). 

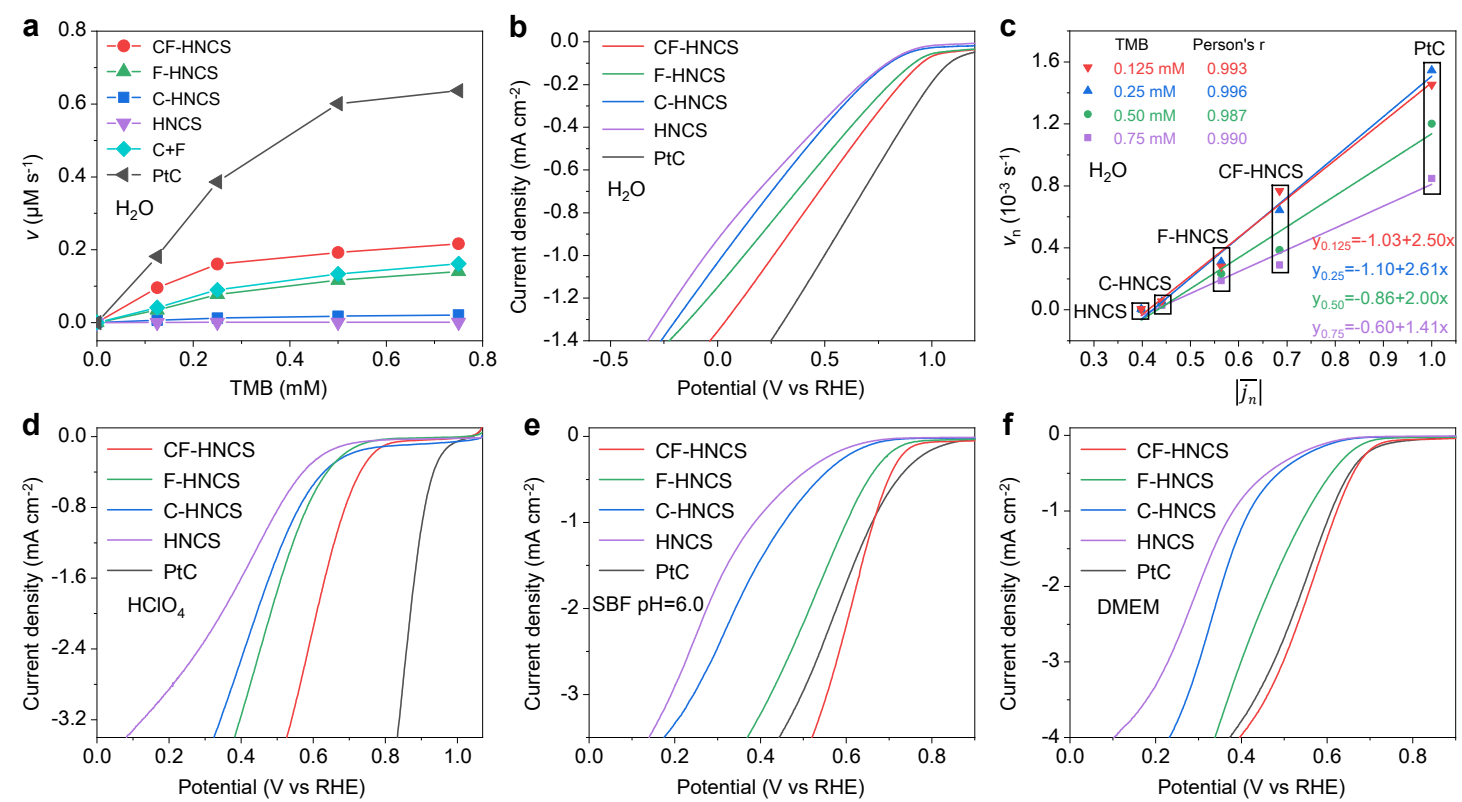

Figure 3. Catalytic performance of CF-HNCS. a, Velocity $(v)$ of the chromogenic reaction in the presence of various samples in deionized water. b, LSV curves of various samples in $\mathrm{O}_{2}$-saturated deionized water. c, The linear fitting between the nondimensionalized velocity $\left(v_{\mathrm{n}}\right)$ of the chromogenic reaction at varied TMB concentrations in deionized water and the absolute value of the normalized mean current density $\left|\overline{j_{n}}\right|$ from 1 to $0 \mathrm{~V}$ in ORR in $\mathrm{O}_{2}$-saturated deionized water. df, The LSV curves of CF-HNCS and reference samples in $\mathrm{O}_{2}$-saturated $0.1 \mathrm{M} \mathrm{HClO}_{4}$ solution (d), $\mathrm{O}_{2}$-saturated SBF solution (e), $\mathrm{O}_{2}$-saturated DMEM solution (f). 

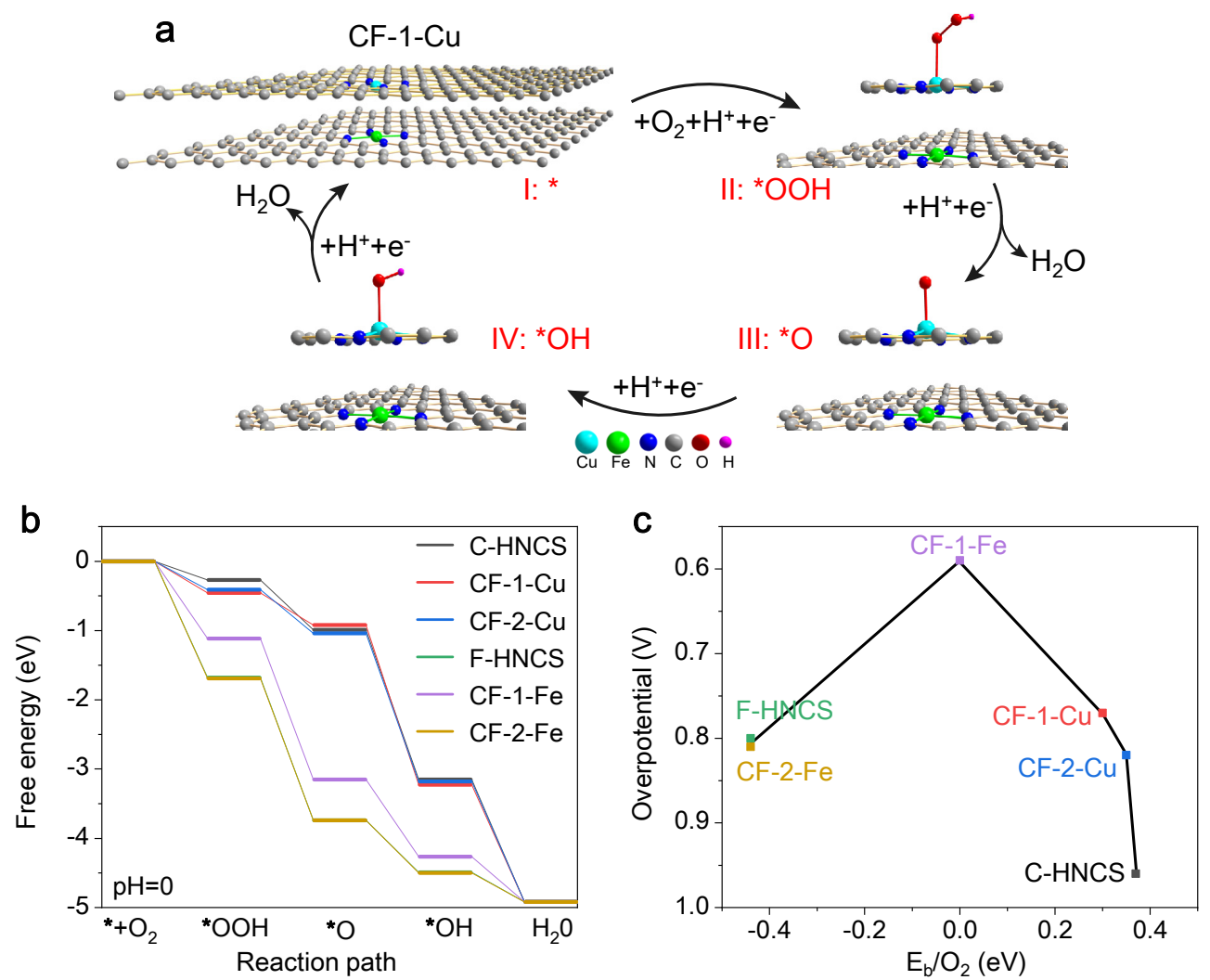

Figure 4. DFT calculations of the Fenton activity. a, Proposed reaction pathways of the ORR on CF-HNCS-1at CF-1-Cu. The grey, dark blue, light blue, green, red, and black balls represent the C, $\mathrm{N}, \mathrm{Cu}, \mathrm{Fe}, \mathrm{O}$, and $\mathrm{H}$ atoms, respectively. b, Free energy profiles of the ORR at different active sites under common conditions ( $\mathrm{U}=0, \mathrm{~T}=298 \mathrm{~K}$ and $\mathrm{P}=1 \mathrm{bar}) . \mathbf{c}$, Thermodynamic relation of the ORR overpotentials with the $\mathrm{O}_{2}$ binding energies at different active sites. 


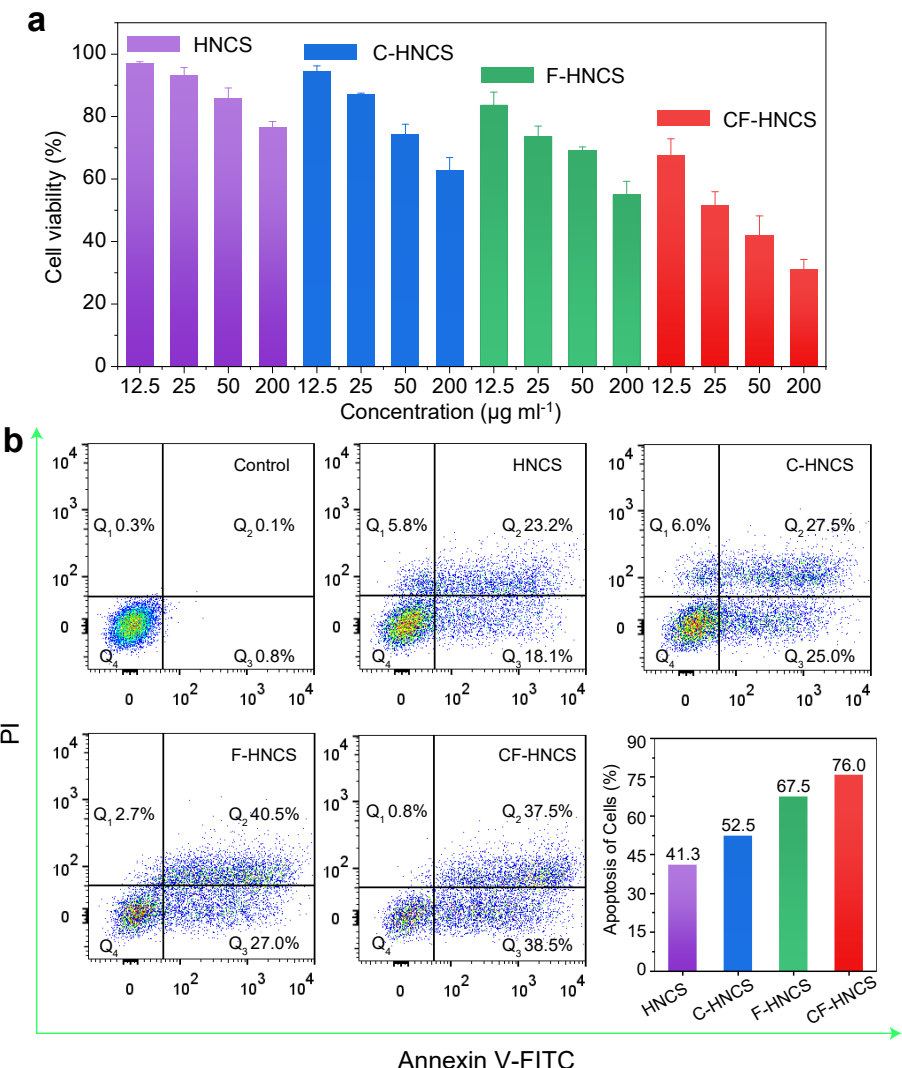

Figure 5. In vitro assessments of the catalytic therapeutic efficacy. a, Viability of $4 \mathrm{~T} 1$ cells after incubation with different concentrations of materials. b, Annexin V-FITC/PI staining analysis of 4T1 cells incubated with different materials (200 ppm). 

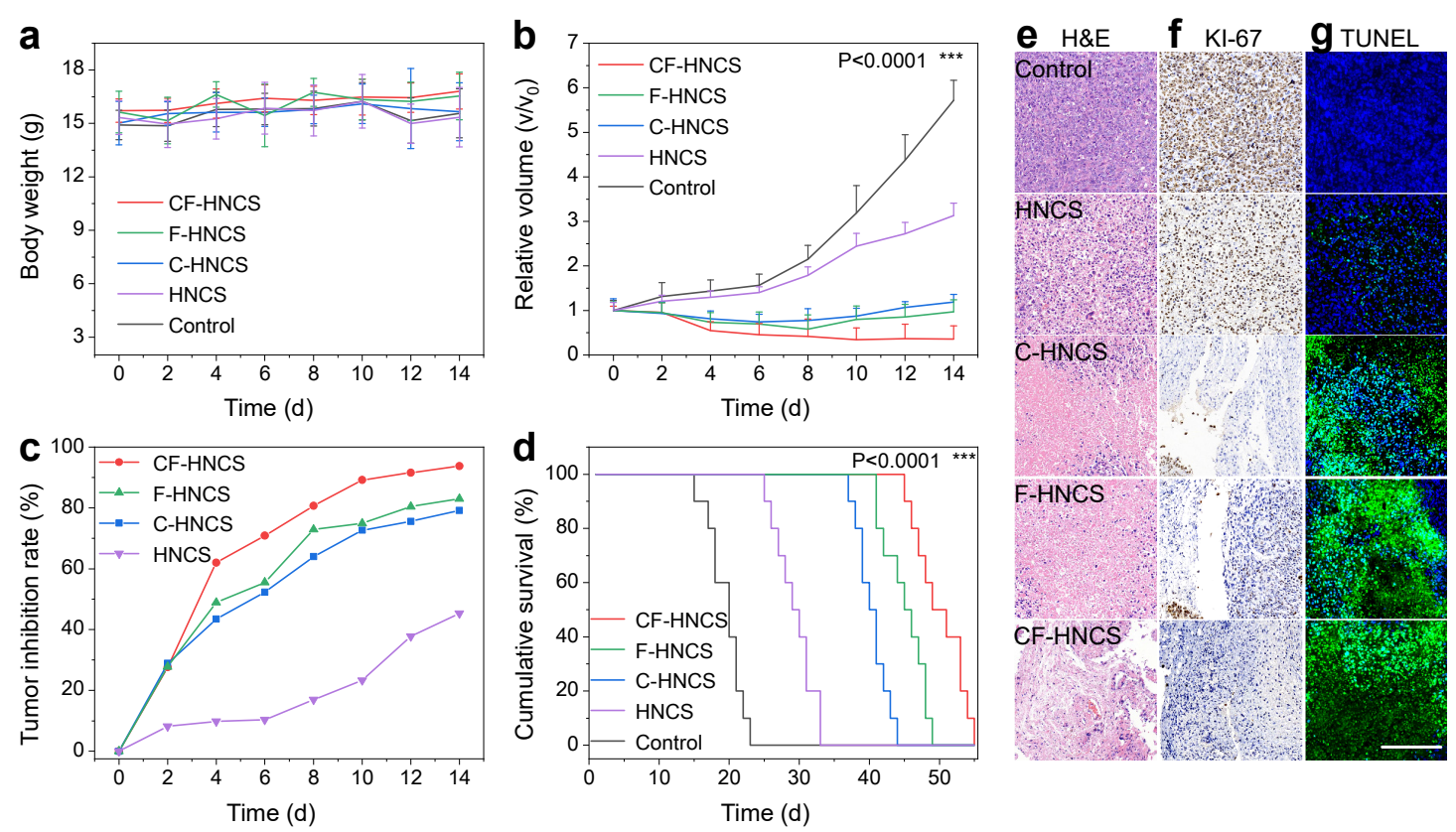

Figure 6. In vivo assessments of the catalytic therapeutic efficacy. a, Schematic illustration of the microneedle administration. b-e, Body weight changes (b), tumour volume changes (c), relative tumour inhibition rates (d), and survival curves (e) of 4T1 xenografted tumour-bearing mice $(\mathrm{n}=$ 10 per group) treated with different catalysts. Data were assessed by Student's two-sided t-test. $* \mathrm{P}<$ $0.1, * * \mathrm{P}<0.05, * * * \mathrm{P}<0.005$ compared to the control group. $\mathbf{f}-\mathbf{h}$, Representative H\&E staining sections for nuclear dissociation (f), Ki-67 immunohistochemical staining sections for cellular proliferation (g) and TUNEL staining sections for necrosis (h) of tumour tissue collected at $14 \mathrm{~d}$ after treatments. Scale bar: $400 \mu \mathrm{m}$. 
Insert Table of Contents artwork here

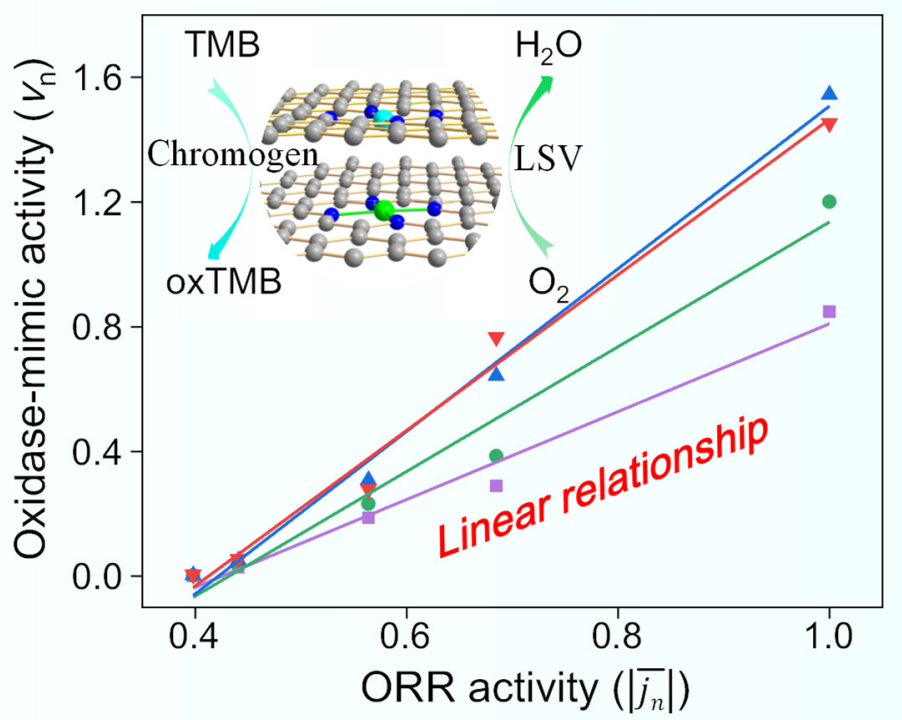

This work reveals the intrinsic homology between oxidase- and electro-catalysis, based on which a facile electrochemical methodology (LSV) has been developed and an effective descriptor $\left(\left|\overline{j_{n}}\right|\right)$ has been proposed for the rapid enzymic catalyst evaluation, which is believed to be inspirable and useful in the design of oxidase-mimics. 